\title{
Valoración de aspectos vinculados al consumo, calidad y seguridad de la carne, en consumidores argentinos de carne
}

\author{
Assessment of aspects related to meat consumption, quality and safety in argentinian \\ meat consumers
}

\author{
Estefanía M. Cáffaro Tommasiello ${ }^{1,2}$; María E. Latorre ${ }^{1,3 *}$; Rosana E. Cepeda ${ }^{4}$ Lorena Garitta $^{3,5}$; \\ Miriam Sosa $a^{3,5}$ y Peter P. Purslow ${ }^{1}$
}

\begin{abstract}
RESUMEN
El crecimiento del sector productor de carne se ve influenciado directamente por los conceptos y criterios de los consumidores. Los aspectos tenidos en cuenta por estos al momento de comprar son numerosos y variados, y los puntos más importantes son la calidad sensorial, higiénico-sanitaria, disponibilidad de productos y tendencia de consumo. Conocer las preferencias, conceptos y actitud de compra por parte de los consumidores permite al sector agroindustrial un mejor crecimiento y desarrollo. El presente trabajo planteó como objetivo estudiar aspectos de consumo, calidad y sanidad de la carne mediante encuesta con consumidores argentinos. Los datos fueron recabados en ciudades de la provincia de Buenos Aires. La encuesta fue anónima, voluntaria y mediante un cuestionario dispuesto on-line. La información obtenida fue analizada mediante test descriptivo según sexo, rango etario, situación laboral y zona de residencia. Los resultados logrados mostraron similitud de comportamiento con otras sociedades latinoamericanas, estudiadas previamente. Los datos indicaron que los rasgos y tendencias más destacados en los hábitos de consumo, criterios de calidad y decisión de compra de carne en ciudadanos de la provincia de Buenos Aires, están relacionados con la edad y en menor medida con el sexo, lugar de residencia o la situación laboral. Las observaciones sugerirían que una buena oportunidad para el sector productor es trabajar en el desarrollo de productos "dirigidos" según los grupos etarios y sus preferencias. En relación con los aspectos de la sanidad, se pudo apreciar que la población usa prácticas en el hogar con elevado potencial de contaminación cruzada y proliferación de microorganismos en los alimentos. Frente a esto se destaca la importancia de los sectores científico y educativo en la necesidad de generar y transmitir información a la sociedad sobre hábitos adecuados de manipulación de alimentos especialmente en hogares.

Palabras clave: productos de origen cárnico, consumidores argentinos, consumo, calidad, sanidad
\end{abstract}

\begin{abstract}
The development of meat producing sector is directly influenced by the concepts and criteria of the consumers. Numerous and diverse aspects are taken into account by consumers at the time of purchase, with appearance, freshness and product availability being the most important points. Knowledge the trends, concepts and willingness to purchase by consumers is allows the agroindustrial sector a better growth and development. The aim at study was to study the consumption, quality and health aspects of meat through a survey of Argentine consumers of meat. The data were collected in cities of the province of Buenos Aires. The survey was anonymous, voluntary and through a questionnaire arranged online. The information collected was analyzed by descriptive test according to: sex, age range, employment status and area of residence. The data indicated that the most outstanding features and trends in consumer habits, quality criteria and decision to purchase meat in citizens of the province of Buenos Aires, are related according to age and, to a lesser extent, sex, residence or employment situation. These observations suggest that would be a good opportunity for the producer's development products orientated to particular age group public and their preferences. Health general aspects suggested it is important for scientific and educational sector generate and transmit more information to society about proper habits of food handling at home.
\end{abstract}

Key words: meat products, argentine consumers, consumption, quality, health

1 Departamento de Tecnología y Calidad de los Alimentos, Facultad de Ciencias Veterinarias, UNCPBA, Tandil, Buenos Aires, Argentina.

2 Comisión de Investigaciones Científicas (CIC), Ministerio de Ciencia, Tecnología e Innovación de la Prov. de Buenos Aires, Argentina. Consejo Nacional de Investigaciones Científicas y Tecnológicas (CONICET), Argentina.

4 Instituto sobre Ecosistemas y Desarrollos Sustentables- CIC- Fac. Cs. Exactas y Fac. Cs. Veterinarias. UNCPBA, Tandil, Argentina.

5 Departamento de Evaluación Sensorial de Alimentos, Instituto Superior Experimental de Tecnología Alimentaria (ISETA), 9 de Julio, Buenos Aires, Argentina.

* Autor por correspondencia: elatorre@unicen.edu.ar; latorre.emilia@gmail.com

Fecha de Recepción: 03 mayo, 2018.

Fecha de Aceptación: 03 agosto, 2018.

DOI: http://dx.doi.org/10.4067/S0718-34292018005001003. Publicado en línea: 6-noviembre-2018. 


\section{Introducción}

La población aumenta y eso trae consigo la demanda de productos alimenticios. Junto a ello, crece el interés del sector productor e industrial de aumentar las ventas e introducir nuevos productos al mercado. A nivel mundial tanto las características referidas a las preferencias y hábitos de consumo, como los tipos de sistemas productivos y el procesamiento de productos cárnicos han ido cambiando a lo largo de las últimas décadas. Datos de la FAO (2009) denotan una importante tasa de crecimiento per-cápita y crecimiento desigual entre los diferentes tipos de carne debido especialmente a la demanda de este producto en países en desarrollo, crecimiento demográfico, urbanización, cambio de hábitos y preferencias.

Hoy conocemos que la calidad de carnes frescas tiene que ver con los atributos que el consumidor busca y valora al momento de comprar. Además sabemos que la carne es un alimento importante para la salud del ser humano y parte significativa del presupuesto de la canasta familiar.

El sector productor e industrial de carne procura aumentar su producción y ventas, y en la inserción en nuevos mercados que demandan sus productos $y / o$ nuevos productos. Hoy el sector agroindustrial conoce la necesidad urgente de aplicar una producción agrícola-ganadera sostenible e integradora y un uso eficiente de los recursos naturales, a fin de adaptarse a los nuevos patrones climáticos y contribuir positivamente cuidando a la sociedad y el medio ambiente (FAO 2015). La producción de alimentos sostenible desempeña un papel clave para lograr un crecimiento integrador a largo plazo, dado que no es una cuestión puramente medioambiental sino que incluye la viabilidad económica y aceptabilidad social (UE, Bruselas, 13.2.2012). El interés de la comunidad científica en comprender y dar respuesta a nuevos interrogantes que plantea la sociedad ha llevado a estudiar el comportamiento de los consumidores.

Varios trabajos latinoamericanos sobre consumo de carne (Albornoz Gotera y Segovia López, 2014; Ngapo, 2014) citan la importancia de los aspectos socioeconómicos y demográficos en los hábitos de consumo, calidad, preferencias e inocuidad de los productos cárnicos.

En Argentina existen numerosos informes sobre el volumen, número de cabezas producidas, faenadas y vendidas, y tipo de carnes volcadas al mercado. Estos datos se encuentran disponibles y en constante cambio y actualización por parte de los distintos sectores (Carne vacuna IPCVA; http://www.ipcva. com.ar; Porcinos A.A.P.P. http://www.porcinos.org. ar/ ; Aviar C.A.P.I.A. http://www.capia.com.ar/ y del ente regulador estatal SENASA http://www.senasa. gob.ar/cadena-animal/porcinos). Sin embargo, pocos estudios se han registrado sobre las características del consumo de carne relacionadas con los aspectos de elección y decisión de compra del consumidor, y conocimientos sobre calidad y seguridad higiénico-sanitaria en el hogar. El desconocimiento e inquietud involucra al sector científico y se abre a la necesidad de estudiar la realidad por parte del consumidor de carne al momento de la decisión de compra y simultáneamente continuar brindando aportes y nuevos conocimientos en los aspectos microbiológicos, físico-químicos, reológicos y tecnológicos.

Así el presente trabajo planteó como objetivo el estudio del consumo de carne mediante encuestas en consumidores de la provincia de Buenos Aires. Y como objetivos específicos: a) estudiar el consumo, calidad y sanidad de la carne; b) analizar las respuestas según las variables sexo, edad, situación laboral y zona de residencia.

\section{Materiales y métodos}

Las encuestas se llevaron adelante utilizando el software Sawtooth versión 8.4.8 (Sawtooth Software Inc., Orem, Utah, USA). Se trabajó con un total de 242 consumidores de carne en ciudades de la provincia de Buenos Aires, durante el período de otoño de 2015. La elección por parte del encuestado fue voluntaria, anónima y mediante sistema on-line. $\mathrm{La}$ encuesta abordó un total de 34 preguntas, las cuales permitieron obtener información sobre el consumo de carne (frecuencia de consumo, tipo de carne, frecuencia de compra, etc.), aspectos vinculados a los conceptos de calidad (criterios y atributos que el consumidor reconoce y/o busca), de sanidad de la carne (hábitos/prácticas de manejo de los productos cárnicos en el hogar) y un conjunto de preguntas finales sobre aspectos/características específicas del consumo de carne vacuna. Las respuestas fueron agrupadas y estudiadas según las variables sexo (femenino y masculino); grupo etario ( $<21$ años; 21 a 30,31 a 40,41 a 50,51 a 60 y > 60 años); situación laboral (Empleado; Ama de casa; Retirado/ jubilado; Estudiante, Ayuda económica del gobierno, Desempleado, Discapacitado y otra) y zona de 
residencia (Zona rural/Pueblo ( $<10.000$ habitantes); Ciudad pequeña (entre 10.000 y 150.000 habitantes); Ciudad intermedia (>150.000 hasta 1.000 .000 de habitantes) y Ciudad grande (>1.000.000 habitantes)).

\section{Análisis estadístico}

Todos los aspectos demográficos fueron vinculados con las variables de cada una de las dimensiones de consumo, sanidad y calidad de la carne, y se analizaron de manera descriptiva mediante el test de asociación de Chi-cuadrado de Pearson. Los valores de Chi-cuadrado de Pearson fueron considerados con un nivel de significancia $(\alpha)$ de 0,05 . Todos los análisis se realizaron con el software libre R (https://www.r-project.org/).

\section{Resultados y discusión}

\section{Características generales de la población bajo estudio}

En la Tabla 1 se presentan las frecuencias relativas de la distribución porcentual (\%) obtenida entre los encuestados para cada una de las variables estudiadas. Tal como muestra la distribución porcentual de las variables (Tabla 1), la encuesta fue atendida principalmente por mujeres. Los rangos etarios se distribuyeron más homogéneamente, sin embargo, los extremos fueron minoritarios. La situación laboral mostró mayoritariamente situación de empleo dentro de los voluntarios ( no se indagó nivel de ingresos) y los residentes pertenecen principalmente a ciudades con una población $\geq 10.000$ y $<1.000 .000$ de habitantes.

Cabe mencionar que la no homogeneidad entre grupos, si bien hubiera sido deseada, no afecta en el estudio de variables descriptivas. A continuación se destacan y describen los resultados de los principales aspectos y aquellos que indican una potencial tendencia o asociación según cada categoría.

\section{Aspectos y valoración de la carne según sexo}

La población encuestada no fue balanceada entre hombres y mujeres, por tal motivo se consideró el total de las respuestas obtenidas para conocer la frecuencia de consumo de las distintas carnes. Observamos (Figura 1) que las carnes aviar $\mathrm{y}$ vacuna fueron las de mayor consumo semanal y las carnes porcina y ovina las de menor consumo. También otros estudios latinoamericanos han encontrado rasgos semejantes, Schnettler et al. (2006) concluyeron que la población de la ciudad de Temuco, en Chile, consume preferentemente carnes bovina $>$ aviar $>$ porcina. Y en su estudio de 2008, Schnettler et al. (2008) observaron que a pesar de que las cifras oficiales indicaran un aparente incremento del consumo de las carnes aviar y porcina, fue nuevamente la vacuna la más frecuentemente consumida y luego la aviar, y que el consumo de carne de cerdo, de pavo y de cordero es ocasional. Tendencias semejantes a las observadas en referencia a la frecuencia de consumo de carne de cerdo fueron obtenidas en Córdoba (Argentina) por García (2005). El autor indica en su trabajo de 2005 que el $50 \%$ de los consumidores de carne no consume carne de cerdo, la frecuencia del consumo de "una vez al mes" representa el 33\% y sólo un 25\% consume con una frecuencia mayor. Por el contrario, Albornoz Gotera y Segovia López (2014), en Venezuela, observaron que el 53\% del total entrevistado consume carne de cerdo.

Tabla 1. Distribución porcentual (\%) de las variables de estudio sobre un total de 242 personas encuestadas.

\begin{tabular}{|c|c|c|c|c|c|c|c|c|}
\hline \multicolumn{2}{|c|}{ Sexo } & \multicolumn{2}{|c|}{$\begin{array}{l}\text { Rango Etario } \\
\text { (años) }\end{array}$} & \multicolumn{3}{|c|}{$\begin{array}{c}\text { Zona de Residencia } \\
\text { (habitantes) }\end{array}$} & \multicolumn{2}{|c|}{ Situación Laboral* } \\
\hline Mujeres & 61,16 & $<21$ & 3,73 & $<10.000$ & & 4,55 & Empleado & 73,55 \\
\hline \multirow[t]{5}{*}{ Hombres } & 38,84 & $21-30$ & 28,63 & 10.000 & 150.000 & 51,24 & Ama de casa & 4,55 \\
\hline & & $31-40$ & 26,56 & 150.000 & - $\quad 1.000 .000$ & 35,54 & Retirado/Jubilado & 8,26 \\
\hline & & $41-50$ & 22,41 & & $>1.000 .000$ & 8,68 & Estudiante & 13,64 \\
\hline & & $51-60$ & 13,69 & & & & & \\
\hline & & $>60$ & 4,98 & & & & & \\
\hline
\end{tabular}




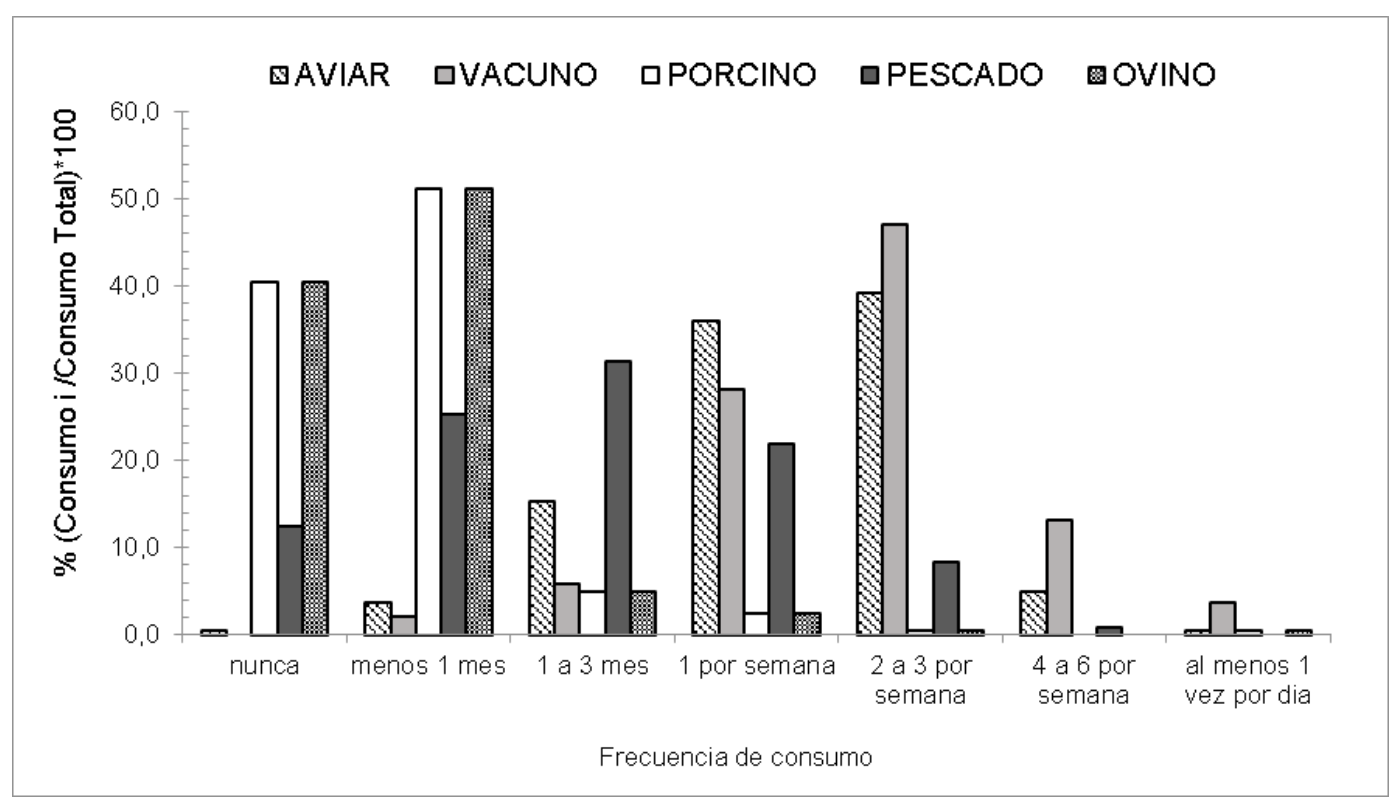

Figura 1. Distribución porcentual de la frecuencia de consumo: distintos tipos de carne para el total de la población encuestada.

El consumo de pescado, tal como se observa en la Fig-1, es de $>1$ vez al mes (1 a 3 veces por mes). El cordero (ovino) mostró también muy bajo consumo (nunca o 1 a 3 menos de una vez por mes). En la encuesta también se consultó sobre el consumo de "otros tipos de carnes" (chivo, vizcacha, pato, conejo, etc.), productos cárnicos y vísceras. Estos datos no se muestran en la Figura 1. En la categoría "otros tipos de carne" la frecuencia de consumo fue nula (nunca) y/o menor a una vez por mes, representando el $85 \%$ y $15 \%$, respectivamente, del total de respuestas. El consumo de productos cárnicos se mostró variado, sin embargo, las opciones 1 a 3 veces por mes y una vez por semana (28 y $27 \%$, respectivamente) fueron las respuestas mayoritarias. El consumo de vísceras fue bajo, siendo la opción nunca (frecuencia nula) la respuesta mayoritaria (63\%) o una menos de una vez por mes (27\%) entre todos los consumidores.

Respecto a la distribución del consumo de las distintas carnes según el período estacional (verano vs invierno), no se encontraron diferencias. En general se consumen indistintamente en verano e invierno (Figura 2). El consumo de productos cárnicos, cordero y pescado es mayor en verano que en invierno.

También se indagó sobre el tipo de cocción empleada en cada período estacional, y se observaron tendencias diferentes entre mujeres y hombres. Así, el uso de parrilla (carne asada, carbón o leña) fue la respuesta predominante en hombres y el uso variado de los tipos de cocción (hervida, parrilla, horno, frita u otra) en mujeres. Considerando el total de respuestas (Figura 3), se concluyó que la cocción a fuego y/o brasas (carne asada/parrilla) tiene preferencia en verano $\approx 48 \%$ o indistintamente en invierno y verano $\approx 41 \%$. Y la cocción a horno mostró mayor empleo en invierno en general. La cocción por inmersión en aceite y/o grasa (freído) mostró un bajo uso: el 70\% de los encuestados dijo no emplearlo nunca (nulo). El $45 \%$ no utiliza la cocción de carne por inmersión en agua (hervido) y el resto de los encuestados mostró variabilidad en la frecuencia de uso según la estación del año. Otros tipos de cocciones (plancha, olla de hierro, etc.) tienen baja aplicación e igual distribución estacional.

En cuanto a criterios de calidad de carne y lo que se está dispuesto a pagar por esta, las respuestas evidenciaron disponibilidad a un pago mayor por frescura predominante en mujeres y por sabor en hombres.

Sobre los conceptos de frescura o vida útil de una carne, si bien se notaron tendencias diferentes entre ambos sexos, en general consideran que el pollo presenta poca vida útil en heladera ( $\leq 1-2$ días), mientras que las carnes de cerdo, vaca y cordero tienen una vida útil mayor, respuestas mayoritarias entre 1-2 días y/o 3-4 días, indistintamente.

En referencia a la temperatura de descongelamiento de las carnes, y si considera que las congeladas deberían 


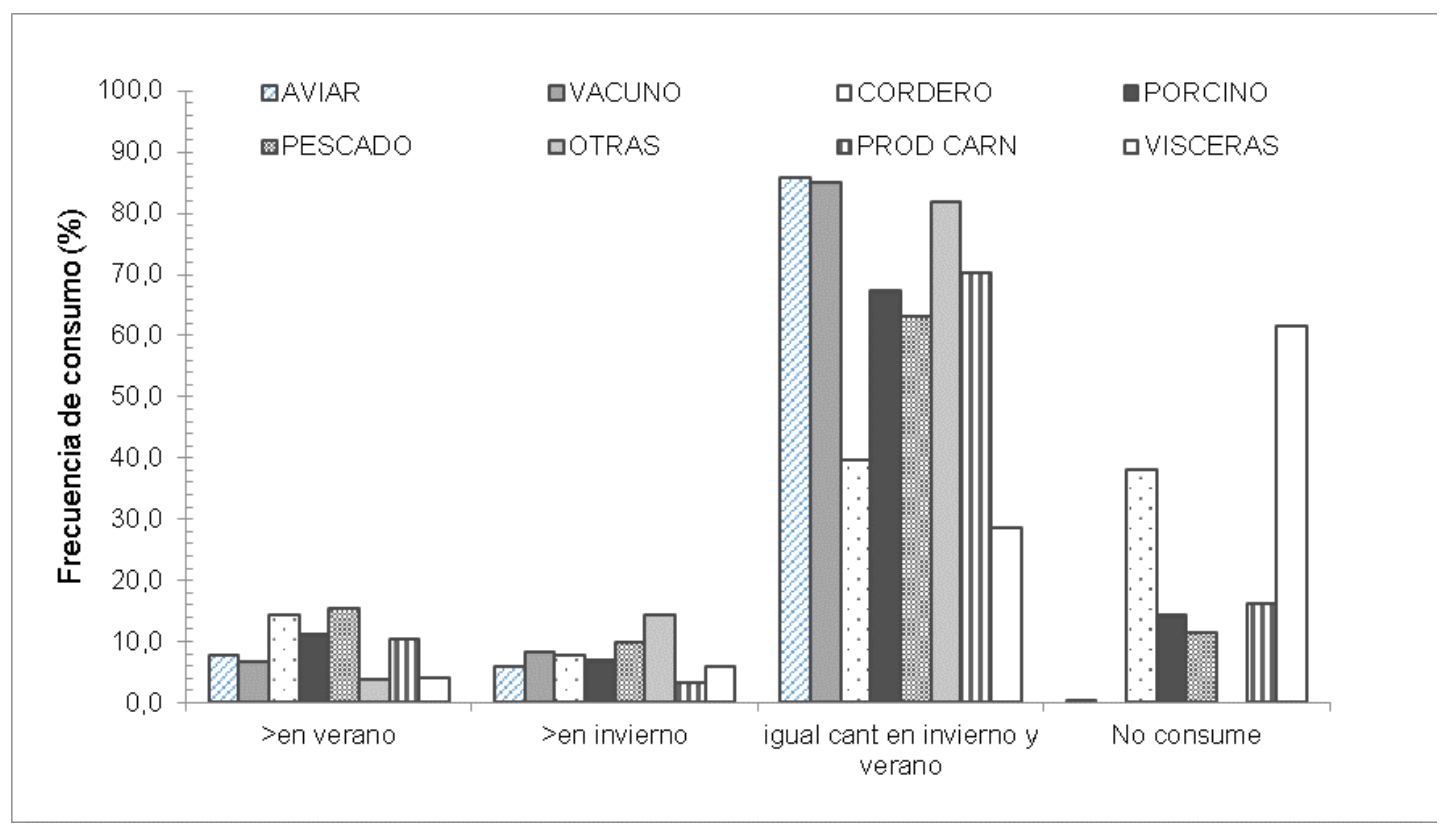

Figura 2. Frecuencia relativa del consumo de los tipos de carne según el período del año, para el total de la población encuestada.

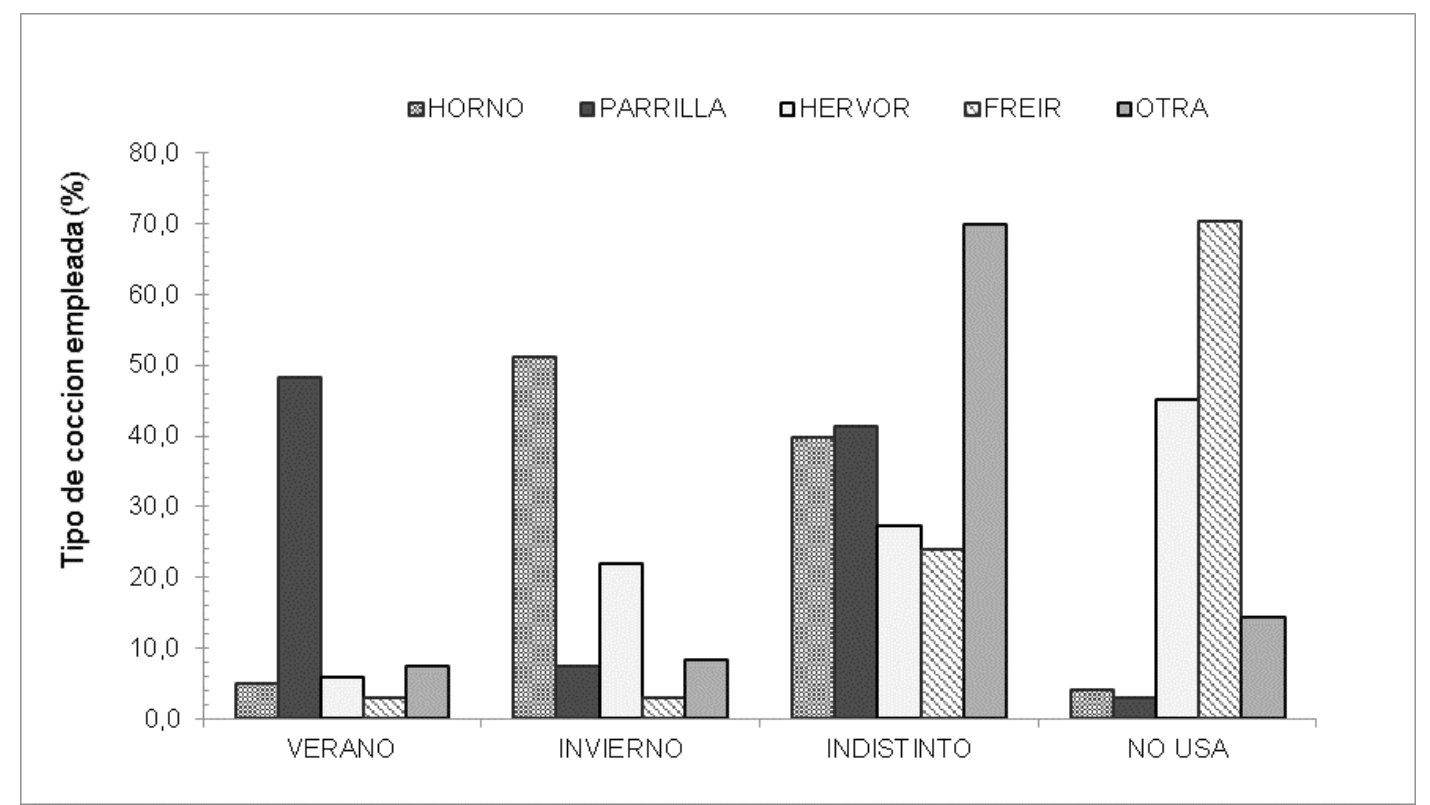

Figura 3. Frecuencia relativa de los tipos de cocción empleada en las carnes según el período del año, para el total de la población encuestada. 
descongelarse siempre en heladera, la respuesta mayoritaria fue sí. Sin embargo, frente a la pregunta: ¿Habitualmente usted dónde descongela la carne congelada? El 65\% de los encuestados aseguró no aplicar el descongelado en heladera. Por otro lado, ante la pregunta: ¿Considera usted que los gérmenes/bacterias que causan enfermedades transmitidas por los alimentos no pueden desarrollarse en la heladera?, un 59,5\% de los encuestados respondió que NO, un $26 \%$ indicó que SÍ es posible el desarrollo de gérmenes/bacterias en la heladera y el resto dijo desconocer este aspecto.

En referencia a los aspectos específicos sobre el consumo de carne vacuna y el proceso de maduración, se efectuó la siguiente pregunta: ¿Usted compraría carne vacuna que desde la faena hasta la venta haya permanecido en el frigorífico durante los tiempos: 1; 2 ; de 3 a 5; de 6 a 10; de 11 a 15 y >15 días? Las opciones posibles para cada tiempo de maduración eran: nunca, rara vez, ocasionalmente, frecuentemente o siempre. Los encuestados argentinos indicaron mayoritariamente la opción nunca sobre la opción $>15$ días. Mientras las respuestas ocasionalmente y frecuentemente fueron para los tiempos 2 días y 3 a 5 días de maduración.

Se les pidió a los encuestados que indiquen las tres clases de carne vacuna (sean cortes, productos elaborados o procesados) que compran con mayor frecuencia y las tres clases que compran con menor frecuencia. Si bien todos señalaron tres clases de carne, la disparidad de respuesta y/o la discordancia de nombres para estas indicarían un bajo conocimiento sobre cortes y usos de cortes de carne vacuna y/o aplicación de distintas formas de despostes entre vendedores.

Resultados similares fueron obtenidos por Schnettler et al. (2006) sobre hábitos de consumo de carne vacuna en Chile, evidenciando un bajo conocimiento en el uso de los cortes en más de un $50 \%$ de los encuestados. También De Andrade (2011), sobre consumidores de carne en Chile, observó con respecto a la carne bovina la poca información disponible sobre los diferentes cortes, en todos los grupos socioeconómicos, y que este factor influye sobre los criterios de calidad y uso del producto.

\section{Aspectos y valoración de la carne según edades}

El análisis de las respuestas sobre los aspectos relacionados con los hábitos de consumo de carnes y los grupos etarios evidenció algunas diferencias. Cabe mencionar que tal como muestra la Tabla 1, los grupos extremos $<21$ años y $>60$ años fueron las categorías con menor número de encuestados. Asimismo, estos grupos presentaron respuestas semejantes a los grupos más próximos. Esto permitió destacar a partir de las observaciones que en relación con la frecuencia de consumo de productos cárnicos (productos pre-elaborados), los adultos mayores (grupos 51 a 60 años y $>60$ años) mostraron un bajo consumo, mientras que los más jóvenes ( $<21$ y de 2130 años) los consumen con mayor frecuencia.

Los resultados obtenidos según consumo y rango etario demostraron también que los adultos mayores (grupos 51 a 60 años y > 60 años) presentan mayor frecuencia de consumo de los productos de la acuicultura que las edades medias (entre 31 a 50 años) y que las categorías jóvenes ( $<21$ y de 21-30 años).

Los aspectos de calidad no presentaron diferencias significativas. Frente a la pregunta: ¿Está usted dispuesto a pagar más por una mejor terneza?, más del $50 \%$ de las respuestas en todas las categorías etarias indicaron que sí lo harían. En cuanto a la valoración de la carne según sabor, terneza, frescura, calidad higiénico-sanitaria, práctica agrícolaganadera ambientalmente sostenible y práctica de bienestar animal, no se observó ninguna tendencia y/o predominancia por alguno de los aspectos. En relación con la calidad de la carne y el sistema productivo empleado en la alimentación del animal, tampoco se mostró ninguna preferencia. Ante la pregunta: ¿En qué medida está de acuerdo con la siguiente afirmación?: La carne de animales criados en feedlot (engorde de corral) es de buena calidad, los consumidores en general se mostraron indistintos/ indiferentes. En cambio el trabajo de Bernués et al. (2003) que evaluó a consumidores de cinco regiones europeas determinó que los consumidores jóvenes dan importancia al sistema productivo empleado, al bienestar de los animales y al medio ambiente. Mientras que los consumidores mayores mostraron una tendencia opuesta. Otro de los puntos que destacan Bernués et al. (2003) es que la sociedad joven europea tiene interés en la información de la etiqueta del producto. Este aspecto, que si bien fue indagado en nuestro estudio, no se vio reflejado dado que mayoritariamente los productos de origen cárnico se obtienen en comercios minoritarios (carnicerías) y no en cadenas de supermercados.

Los conceptos referidos a sanidad (hábitos en el hogar) considerando los grupos de edades arrojaron rasgos de interés. Por un lado, frente a la pregunta: ¿Usted dónde guarda con mayor 
frecuencia la carne cruda?, la respuesta: en la parte alta (bandeja superior) de la heladera fue la predominante en los grupos $<21$ años, 21 a 30 y 31 a 40 años. Las personas de entre $41-50$ y 51- 60 mostraron dispersión en las respuestas (\% equivalentes 25-30 en las respuestas bandeja alta, baja, medio y/o donde haya lugar).

Si bien sabemos que el número de personas encuestadas jóvenes < 21 años fue pequeño con relación a los demás grupos etarios, los datos recolectados demostraron no saber si la carne picada es más propensa a los gérmenes/ contaminación que otros cortes. Este grupo también respondió: "Cortar otro alimento que estaba cocido o listo para consumir, utilizando la misma tabla y cuchillo" frente a la pregunta: La última vez que cortó carne cruda, ¿qué fue lo que usted hizo inmediatamente después con el utensilio utilizado? A partir de las respuestas de este grupo (<21 años) junto con las del grupo 21 a 30 años, en referencia a los hábitos de manejo de carnes en el hogar, se observan indicios de carencias en cuanto a los conceptos sobre contaminación cruzada de alimentos.

El trabajo de Gutiérrez et al. (2015) estudió las conductas de los consumidores de Neiva (Colombia) en relación con el manejo de los alimentos durante la compra, preparación y almacenamiento en el hogar, debido a que los hábitos inherentes a dichas prácticas pueden delimitar (disminuir o aumentar) el nivel de riesgo al que se exponen. Los autores observaron que el $43 \%$ de las personas utilizan la misma superficie para cortar y solo se lava al final del trabajo. Dicha práctica implica un riesgo potencial elevado para la salud asociado a la contaminación cruzada de los alimentos, especialmente al manipular carnes y verduras de manera conjunta. Gutiérrez et al. (2015) constataron la existencia de un elevado porcentaje de hábitos que presentan deficiencias en seguridad alimentaria en los hogares. También observaron que aquellos consumidores con conductas que aseguran de cierta forma la inocuidad presentaron falencias en puntos que pueden revestir peligro para la salud. Los resultados aquí obtenidos en consumidores argentinos frente a las conductas relacionadas con la manipulación de productos cárnicos en el hogar y el potencial asociado a la contaminación cruzada de alimentos reflejan carencias de conocimientos y evidencian la necesidad de brindar información sobre este tema.
Aspectos y valoración de la carne según situación laboral

En relación con las respuestas obtenidas en los grupos mayoritarios (empleado; estudiantes; retirado/ jubilado; ama de casa), se observó que el consumo de carne vacuna, aviar y porcina no presenta diferencias según la situación laboral. En cuanto a los aspectos relacionados con la calidad de la carne, y ante las preguntas: ¿Considera usted que la carne congelada en el hogar es de buena calidad? y ¿Considera usted que la carne de carnicería es de buena calidad?, el grupo amas de casa aseguró estar "totalmente de acuerdo" con ambas premisas. Sin embargo, los estudiantes consideran que "la carne congelada en el hogar" no es de buena calidad, como tampoco lo es la de carnicería.

Con respecto a la sanidad de la carne, los empleados, las amas de casa y los estudiantes indicaron las opciones descongelado de la carne en heladera, a temperatura ambiente en la mesada o bacha de la cocina con agua caliente. Frente a la pregunta: Si usted tiene sobras de carne cocida en la heladera, pero no recuerda cuánto hace que están allí, ¿cómo determina si son seguras o no para comerlas?, las amas de casa y los empleadosseñalaron principalmente las opciones "oler y mirar la misma" o "las tiran debido a no recordar el tiempo que hace que están en la heladera”. Sobre los demás aspectos vinculados a la sanidad de la carne no se vieron rasgos y/o predominancia de ningún grupo.

\section{Aspectos y valoración de la carne según zona de residencia}

Si bien los grupos no fueron balanceados para esta categoría (Tabla 1), de la observación de las respuestas se concluyó que las zonas de residencia podrían mostrar tendencias en cuanto a los aspectos relacionados con el consumo de carne. Un dato notorio fue la frecuencia del consumo de cordero en esta región (provincia de Buenos Aires, Argentina), donde se observó que la población de la zona rural $(<10.000$ habitantes) indicó una frecuencia de consumo 1 a 3 veces por mes, mientras que las zonas restantes $(\geq$ 10.000 habitantes) dijeron hacerlo menos de una vez por mes y/o nunca. Esto es esperable para esta región donde la producción de ovinos es pequeña y se da principalmente en zonas rurales para el consumo personal/familiar y no como sistema productivo, a diferencia de otras provincias argentinas. Los 
aspectos vinculados a calidad y sanidad de la carne no mostraron rasgos de predominancia y/o tendencias.

\section{Conclusiones}

El consumo de carne vacuna y aviar predomina sobre la porcina, pescado, ovino y otras. Entre los tipos de cocción de carnes, los elegidos principalmente son el horno y la parrilla, mientras que la fritura no es empleada con frecuencia. Observamos además que actualmente el concepto maduración y calidad de carne no es aún conocido por los consumidores argentinos. Algunos grupos etarios mostraron tendencias diferentes entre frecuencia de consumo del tipo de carne y edad, especialmente entre los grupos más jóvenes $<30$ años y los grupos de adultos mayores a $>50$ años. Con respecto a la situación laboral se observaron tendencias de comportamiento diferentes entre estudiantes y amas de casa. Lo mismo ocurrió con las zonas de residencia, donde la zona rural manifestó consumir más cordero que las demás. Los resultados aquí obtenidos mostraron similitud de comportamiento con otras sociedades latinoamericanas estudiadas por otros autores, y es el factor socioeconómico-demográfico en su conjunto lo que influye en las características de consumo y conceptos de calidad y sanidad de la carne. También se advirtieron carencias en los criterios de buenas prácticas-hábitos de manipulación de alimentos en el hogar, con especial referencia a las carnes.

Los rasgos y tendencias observados indicarían que sería una buena oportunidad para el sector productor trabajar en la producción y el desarrollo de productos en pos de un público en particular, y orientados principalmente a los distintos grupos etarios según sus preferencias. En referencia a los aspectos de la sanidad de las carnes en general, las respuestas obtenidas demuestran la importancia de generar y transmitir, desde los sectores científico y educativo, una mayor información a la sociedad sobre buenos hábitos de manipulación de alimentos, conceptos nutricionales de las distintas carnes y características de uso de los cortes musculares, especialmente vacunos.

\section{Agradecimientos}

Los autores agradecen el apoyo financiero de CONICET, a la Universidad Nacional de Centro de la Provincia de Buenos Aires (UNCPBA) y al Instituto Superior Experimental de Tecnología de los Alimentos (ISETA).

\section{Literatura citada}

Albornoz Gotera, A. J.; Segovia López, E. M.

2014. Hábitos de compra-consumo de la carne fresca de cerdo en Maracaibo, estado Zulia-Venezuela Purchase - consumption habits of fresh pork meat in Maracaibo, Zulia State- Venezuela. Zootecnia Trop., 32 (2): 169-177.

Bernués, A.; Olaizola, A.; Corcoran, K.

2003. Extrinsic attributes of red meat as indicators of quality in Europe: An application for market segmentation. Food Quality and Preference, 14: 265-276.

Comunicación de la Comisión al Parlamento Europeo, al Consejo, al Comité Económico y Social Europeo y al Comité de las Regiones.

2012. La innovación al servicio del crecimiento sostenible: una bioeconomía para Europa. Bruselas, 13.2.2012 COM (2012) 60p.

FAO.

2009. El estado mundial de la agricultura y la alimentación - La ganadería, a examen. Roma: Organización de las Naciones Unidas para la Alimentación y la Agricultura (FAO) $184 \mathrm{p}$.

FAO.

2015. Organización de las Naciones Unidas para la Alimentación y la Agricultura, Roma. 52p.
García, S.D.

2005. Caracterización del consumo de carne porcina en la ciudad de Córdoba-Argentina. Facultad de Ciencias Agropecuarias Universidad Nacional de Córdoba. Córdoba, Argentina. 7p.

Gutiérrez, N.; Durango, S.G.; Carrillo, N.J.

2015. Hábitos en seguridad alimentaria de consumidores en Neiva. Food safety habits in consumers in Neiva. Revista Ingeniería y Región, 13 (1): 25-35.

Ngapo, T.

2014. Aplicación de la ciencia de consumidores en la investigación y desarrollo de la carne. Consumer Science applications in meat research and development. NACAMEH 8 (1): S43-S52.

Schnettler, M.B.; Manquilef Baschler, O.; Miranda Vargas, H. 2006. Hábitos de consumo de carne bovina en Temuco, IX región de Chile. Idesia, 24 (2): 15-23.

Schnettler, M.B.; Silva, E.R.; Sepúlveda, B.N.

2008. Consumo de carne en el sur de Chile y su relación con las características sociodemográficas de los consumidores. Revista Chilena de Nutrición, 35 (1): 262-270. https://dx.doi. org/10.4067/S0717-75182008000400002

Segovia, E.; Contreras, D.; Marcano, D.; Pirela, R.; Albornoz, A. 2005. Conducta del consumidor de carne bovina según clase socioeconómica en el municipio Maracaibo, estado Zulia, Venezuela. Agroalimentaria, 10 (21): 113-121. 